\title{
CONTINUOUS IMAGES OF LUSIN SETS
}

\author{
J. B. BROWN AND G. V. COX
}

\begin{abstract}
It is the purpose of this note to further clarify the relationship between continuous images of Lusin sets and concentrated sets.
\end{abstract}

We are concerned with subsets of separable metric spaces which have singularity properties such as those discussed in $\S 40$ of Kuratowski's Topology [3] (also see [1] for a more complete survey). These are the sets which are "small" in either a Baire categoric sense ("rarified sets" and "always first category" sets) or a measure theoretic sense. We are concerned with the latter type. In particular, consider the following properties of subsets $M$ of a separable metric space $X$.

$L($ rel $X)$ : every nowhere dense subset of $X$ intersects $M$ in an at most countable set,

$\nu: M$ has property $L(\operatorname{rel} M)$,

$L_{1}: M$ is the union of countably many $\nu$ sets,

concentrated about a set $C: C \subseteq X$ and every open set containing $C$ contains all but at most countably many elements of $M$,

con (rel $X): M$ is concentrated about a countable subset of $X$,

$P: M$ is con $($ rel $M)$,

$C^{\prime \prime}$ : for every system $\{G(x, n) \mid x \in X, n=12, \ldots\}$ of open sets such that $x \in$ $G(x, n)$ for each $x$ and $n$, there necessarily exists a diagonal sequence $\left\{x_{n}\right\}$ of elements of $X$ such that $X \subseteq G\left(x_{1}, 1\right) \cup G\left(x_{2}, 2\right) \cup \cdots$.

$C($ rel $\delta): \delta$ is a metric compatible with the topology of $X$ and for every sequence $\left\{\varepsilon_{n}\right\}$ of positive numbers, there exists a sequence $\left\{x_{n}\right\}$ of elements of $X$ such that $X \subseteq N_{\delta}\left(x_{1}, \varepsilon_{1}\right) \cup N_{\delta}\left(x_{2}, \varepsilon_{2}\right) \cup \cdots$, where $N_{\delta}(x, \varepsilon)$ denotes the $\varepsilon$-nbhd of $x$ under the metric $\delta$.

$\beta$ : for every complete continuous measure $\mu$ on $M, \mu(M)=0$.

It is well known that for subsets of a separable metric space the following implications hold:

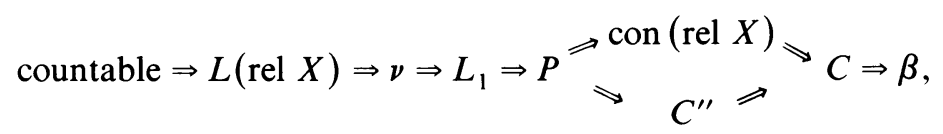

and that if the Continuum Hypothesis $(\mathrm{CH})$ is assumed all the possible implications not implied by the above diagram fail to hold.

Received by the editors October 29, 1982 and, in revised form, January 10, 1983.

1980 Mathematics Subject Classification. Primary 28A05; Secondary 54H05.

Key words and phrases. Lusin sets, concentrated sets.

(C)1983 American Mathematical Society 0002-9939/82/0000-1436/\$01.75 
There has been a great deal of study about what happens to sets with the above properties under various kinds of transformations. It is shown in [3] that the continuous image of a $\nu$ set has property $C^{\prime \prime}$. It is shown in [2] that the image of a $\nu$ set under a Borel function must have property $\beta$. It was pointed out in [5] that the image of a $\nu$ set under a function which has the property of Baire (in the weak sense)

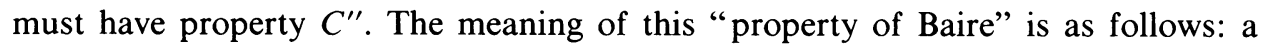
subset $M$ of a space $X$ has property $B_{w}($ rel $X)$ if there exist an open subset $Q$ of $X$ such that $M-Q$ and $Q-M$ are of first category. Then $n$ function $f$ is said to have property $B_{w}$ if for every closed subset $P$ of the range of $f, f^{-1}(P)$ has property $B_{w}$ (rel domain of $f$ ). Of course, continuous functions are Borel functions, and Borel functions are $B_{w}$ functions. First, we show that all of the above discussed images are the same type of set.

THEOREM. If $Z$ is the $B_{w}$-image of a $\nu$ set, then $Z$ is actually the continuous 1-1 image of a set with property $L($ rel the reals $R$ ).

Proof. Let $X$ be a $\nu$ space and $f: X \rightarrow Z$ a $B_{w}$ function. It follows [3, p. 400] that there is a first category subset $P$ of $X$ such that $g=f \mid(X-P)$ is continuous. $P$ is countable. Now, construct a space $Y$ that contains $(X-P)$ isometrically and such that $Y-(X-P)$ is countable and discrete. Construct a function $h$ from $Y$ onto $Z$ such that $h \mid(X-P)$ is $g$. Then $Y$ is also a $\nu$ space and $h$ is a continuous function from $Y$ onto $Z$. Now, consider a subset $Y^{\prime}$ of $Y$ such that $h^{\prime}=h \mid Y^{\prime}$ is $1-1$ and continuous from $Y^{\prime}$ onto $Z$. $Y^{\prime}$ is also a $\nu$ space. We know from [4] that there is a set $M$ of real numbers with property $L\left(\right.$ rel $R$ ) and a homeomorphism $t$ from $M$ onto $Y^{\prime}$. Then the composition $t \circ h^{\prime}$ is a 1-1 continuous function from $M$ onto $Z$.

From now on, we will say that a set which is the continuous image of a $\nu$ set has property $C(\nu)$. Thus, we know that $C(\nu)$ implies $C^{\prime \prime}$. We will see below that $C(\nu)$ implies $P$ and even a hereditary version of $P$. In one of the most beautiful constructions given in this whole area of research, Rothberger [6] showed that $\mathrm{CH}$ implies the existence of a subset $M$ of the irrationals such that $M$ is concentrated about the rationals $Q$, but $M$ is concentrated about no countable subset of itself. It follows that $M \cup Q$ satisfies property $P$, but not property $H P$ (every subset has property $P$ ). We now fit properties $C(\nu)$ and $H P$ into the previous diagram of implications.

THEOREM. $L_{1} \Rightarrow C(\nu) \Rightarrow H P \Rightarrow P$.

Proof. Suppose $X$ is the union $X_{1} \cup X_{2} \cup \cdots$ of $\nu$ spaces. We may assume the $X_{i}$ 's are disjoint. Consider a space $Y=X_{1}^{\prime} \cup X_{2}^{\prime} \cup \cdots$, where for each $i, X_{i}^{\prime}$ is an isometric image of $X_{i}$, but the $X_{i}^{\prime}$ are not only disjoint but mutually separated. Then, $Y$ is a $\nu$ space and the function $f$ from $Y$ onto $X$ which identifies the elements of $X_{i}^{\prime}$ with the elements of $X_{i}$ in the natural way is continuous. Thus, $L_{1} \Rightarrow C(\nu)$.

Now, suppose $X$ is a $\nu$ space and $f$ is a continuous function from $X$ onto $Y$. We first show $Y$ has property $P$. Let $C$ be a countable subset of $Y$ such that $f^{-1}(C)$ is dense in $X$. Let $Q$ be an open subset of $Y$ that contains $C$. It follows that $f^{-1}(Q)$ is an open subset of $X$ which is dense in $X$. Thus, $X-f^{-1}(Q)$ is nowhere dense and 
therefore countable. It follows that $Y-Q$ is countable so that $Y$ has property $P$. Since property $\nu$ is a hereditary property it actually follows that $C(\nu) \Rightarrow H P$.

EXAMPLE $(\mathrm{CH}): L_{1} \Leftarrow C(\nu)$. For each irrational number $x$ in the interval $[0,1]$, let $T(x)=\left\{n_{i}\right\}$ be the sequence of positive integers in the continued fraction expansion of $x$. Let $K(1), K(2), \ldots$ be a sequence of disjoint Cantor subsets of $[0,1]$ with diameters $<1$ such that if $U$ is open in [0,1], $U$ contains one of the $K(i)$. For each $i$, let $K(i, 1), K(i, 2), \ldots$ be a sequence of disjoint Cantor subsets of $K(i)$ with diameters $<\left(\frac{1}{2}\right)^{i}$ such that if $U$ is open relative to $K(i), U$ contains one of the $K(i, j)$. Continue this process. We say that a set $A$ is uncountably (categorically) dense in a set $B$ if every open subset of $B$ intersects $A$ in a set which is uncountable (2nd category relative to $B$ ). Now, assume $\mathrm{CH}$ and let $X$ be a $\nu$ space which is a subset of and uncountably dense in the irrationals (see [3, p. 525]). Let $f$ be the function with domain $X$ such that if $x \in X$ and $T(x)=\left\{n_{i}\right\}$, then $f(x)$ is the point common to $K\left(n_{1}\right), K\left(n_{1}, n_{2}\right), K\left(n_{1}, n_{2}, n_{3}\right), \ldots$ Clearly, $f$ is $1-1$ and continuous, so $Y=f(X)$ is a $C(\nu)$ space. Now suppose that $Y=Y_{1} \cup Y_{2} \cup Y_{3} \cup \cdots$, where each $Y_{i}$ is a $\nu$ space. One of the $Y_{i}$ must be uncountable, assume it is $Y_{1}$. Then $f^{-1}\left(Y_{1}\right)$ is uncountable and since $X$ is a $\nu$ space, it follows that $X_{1}=f^{-1}\left(Y_{1}\right)$ is second category. It follows that there is a finite sequence $i_{1}, i_{2}, \ldots, i_{k}$ of positive integers such that if $(a, b)$ is the open interval with intersection with the irrationals consisting of those irrationals which have $i_{1}, \ldots, i_{k}$ as the first $k$ terms of $T(x)$, then $X_{1}$ is categorically dense, and therefore uncountably dense in $(a, b)$. It follows that $f\left(X_{1} \cap(a, b)\right)$ is uncountably dense in $f(X \cap(a, b))$. Since

$$
f\left(X_{1} \cap(a, b)\right) \subseteq Y_{1} \text { and } f(X \cap(a, b))=Y \cap K\left(i_{1}, \ldots, i_{k}\right),
$$

we have that $Y_{1}$ is uncountably dense in $Y \cap K\left(i_{1}, \ldots, i_{k}\right)$. There exists a positive integer $m$ such that $Y_{1} \cap K\left(i_{1}, \ldots, i_{k}, m\right)$ is uncountable, but this will be nowhere dense relative to $Y_{1}$, which contradicts the assumption that $Y_{1}$ is a $\nu$ space.

Problem. We have been unable to determine whether or not $C(\nu) \Leftarrow H P$, and leave that as an open problem.

ADDED IN PROOF. M. N. Lusin showed in [Fund. Math. 21 (1933), 114-128] that the space $Y$ of the above example is always first category.

\section{REFERENCES}

1. J. B. Brown and G. V. Cox, Classical theory of totally imperfect sets, Real Analysis Exchange 7 (1982). $185-232$.

2. R. B. Darst, properties of I.usin sets with applications to prohability, Proc. Amer. Math. Soc. 20 (1969), $348-350$.

3. C. Kuratowski, Topologv. Vol. I, Academic Press, New York, 1966.

4. C. Kuratowski and W. Sierpinski, Sur les ensembles qui ne contiennent aucun sous-ensemble indenombrable non-dense, Fund. Math. 26 (1936), 137.

5. F. Rothberger, Eine vershärfung der eigenshaft $C$, Fund. Math. 30 (1938), 50-55.

6. Sur les familles indenombrables de suites de nombres naturels et les problemes concernant la propriété C, Proc. Cambridge Philos. Soc. 37 (1941), 109-126.

Department of Mathematics, Auburn University, Auburn, Alabama 36849

Tracor, Inc., Austin, TeXas 78721 